\title{
Effects of patient age and choice of antisecretory agent on success of eradication therapy for Helicobacter pylori infection
}

\author{
Toshihiro Nishizawa, ${ }^{1,2,3}$ Hidekazu Suzuki, ${ }^{4, *}$ Ai Fujimoto, ${ }^{1,2}$ Hiroto Kinoshita, ${ }^{1,5}$ Shuntaro Yoshida, ${ }^{1,5}$ \\ Yoshihiro Isomura, ${ }^{1,6}$ Akira Toyoshima, ${ }^{1,7}$ Takanori Kanai, $^{3}$ Naohisa Yahagi ${ }^{2}$ and Osamu Toyoshima ${ }^{1}$ \\ ${ }^{1}$ Gastroenterology, Toyoshima Endoscopy Clinic, Tokyo 157-0066, Japan \\ ${ }^{2}$ Division of Research and Development for Minimally Invasive Treatment, Cancer Center, ${ }^{3}$ Division of Gastroenterology and Hepatology, \\ Department of Internal Medicine and ${ }^{4}$ Medical Education Center, Keio University School of Medicine, 35 Shinanomachi, Shinjuku, Tokyo 160-8582, Japan \\ ${ }^{5}$ Department of Gastroenterology, Graduate School of Medicine, The University of Tokyo, Tokyo 113-0033, Japan \\ ${ }^{6}$ Department of Gastroenterology, Kanto Central Hospital, Tokyo 158-8531, Japan \\ ${ }^{7}$ Department of Surgery, Japanse Red Cross Medical Center, Tokyo 150-8935, Japan
}

(Received 21 September, 2016; Accepted 14 November, 2016; Published online 16 February, 2017)

\begin{abstract}
The effects of patient age on the efficacy of eradication treatment for Helicobacter pylori $(H$. pylori) remain unclear. The present study aimed to determine whether age affects eradication therapy involving vonoprazan, a novel potassium-competitive acid blocker (PCAB). We reviewed the cases of 3,261 patients who were administered first-line and second-line $H$. pylori eradication therapy at Toyoshima Endoscopy Clinic. The first-line treatment was clarithromycin and amoxicillin combined with a proton pump inhibitor (PPI) or a PCAB. The second-line treatment was metronidazole and amoxicillin combined with a PPI or PCAB. The patients were divided into a young to middle-aged group (age $\leq 50$ years) and an older group (age $>50$ years) as well as into PPI and PCAB groups. The PPI-clarithromycin-amoxicillin regimen demonstrated a significantly lower $\boldsymbol{H}$. pylori eradication rate than the PCAB-clarithromycinamoxicillin regimen $(p<0.001)$. With the PPI-clarithromycin-amoxicillin regimen, the eradication rate in the young to middle-aged group was significantly lower than that in the older group $(p<0.001)$. Lastly, age had no impact on the eradication rate of PCAB-based therapy or metronidazole-based therapy. In conclusion, with clarithromycin-based triple therapy, PCAB is a better choice of antisecretory agent compared to PPIs, especially in young to middle-aged patients.
\end{abstract}

Key Words: H. pylori, eradication, age, vonoprazan, proton pump inhibitor

E radication of Helicobacter pylori (H. pylori) infection has been reported as an effective strategy in the treatment of peptic ulcers and gastric mucosa-associated lymphoid tissue lymphoma as well as in the prevention of gastric cancer. ${ }^{(1-6)}$ The first-line eradication regimen of clarithromycin combined with amoxicillin and a proton pump inhibitor (PPI) is covered under national health insurance in Japan. ${ }^{(7)}$ However, the success rate of this regimen has recently dropped to $\leq 75 \%$ because of an increasing incidence of clarithromycin resistance. ${ }^{(8-10)}$ The secondline regimen, using metronidazole combined with amoxicillin and a PPI, is also covered under national health insurance in Japan, ${ }^{(11)}$ and the prevalence of $H$. pylori resistance to metronidazole in Japan is $5-12 \%$, while the success rate of this second-line regimen has remained constant at approximately $90 \%$. $^{(12,13)}$

In 2015, vonoprazan, a member of a new class of potassiumcompetitive acid blockers (PCABs), was released and approved for use in $H$. pylori eradication in Japan. Murakami et al. ${ }^{(14)}$ have reported the superiority of vonoprazan over lansoprazole for first-line $H$. pylori eradication therapy, with an eradication rate of $93 \%$ for vonoprazan and $76 \%$ with lansoprazole $(p<0.01)$. Mamori et $a l .{ }^{(15)}$ reported that first-line eradication failures occurred more frequently in patients aged less than 50 years than in those aged over 50. However, it remains unknown whether patient age affects PCAB-based therapy and metronidazole-based treatment. The aim of the present study was to determine whether age affects the eradication of $H$. pylori in routine clinical practice.

\section{Methods}

Subjects. We retrospectively reviewed the data of 3,261 patients who were administered first- and second-line $H$. pylori eradication therapy at the Toyoshima Endoscopy Clinic between February 2002 and June 2016. H. pylori positivity in these patients was confirmed from the results of the ${ }^{13} \mathrm{C}$-urea breath test, stool antigen test, or the presence of $H$. pylori-specific IgG antibodies in the serum. ${ }^{(16)}$

First-line triple therapy included $200 \mathrm{mg}$ clarithromycin, $750 \mathrm{mg}$ amoxicillin, and an antisecretory agent (30 mg lansoprazole, $10 \mathrm{mg}$ rabeprazole, or $20 \mathrm{mg}$ vonoprazan) twice daily for 1 week. Eradication was confirmed using the urea breath test at least 4 weeks after the treatment. The cut-off value for the urea breath test was $2.5 \%$. Patients in whom first-line therapy failed to eradicate the pathogen received second-line triple therapy with $250 \mathrm{mg}$ metronidazole, $750 \mathrm{mg}$ amoxicillin, and an antisecretory agent $(30 \mathrm{mg}$ lansoprazole, $10 \mathrm{mg}$ rabeprazole, or $20 \mathrm{mg}$ vonoprazan) twice daily for 1 week. The antisecretory agent was chosen by the physician in charge. Favorable eradication data for vonoprazan were released in February 2015, and this has been the antisecretory agent of choice since March 2015.

The success rates of eradication were assessed using intentionto-treat (ITT) and per protocol (PP) analyses. Patients who did not return to the clinic to receive a urea breath test for evaluating the results of eradication therapy were excluded from the PP analysis.

Statistical analysis. Patients were classified into a young to middle-aged group (age $\leq 50$ years) and an older group (age $>50$ years) to examine the effect of age on treatment success. Further, patients were also classified into the PPI (lansoprazole or rabeprazole) and PCAB group (vonoprazan) to examine the effect of antisecretory agent on treatment success.

*To whom correspondence should be addressed.

E-mail: hsuzuki.a6@keio.jp 
Differences between the groups were compared using the chisquared test for categorical variables. A $p$ value of less than 0.05 was considered statistically significant. Data were analyzed using the Stat Mate IV software (ATOMS, Tokyo, Japan).

Ethics. The study was approved by an external ethics committee, and informed consent was obtained from all patients. The University Hospital Medical Information Network clinical trial registration number is UMIN000018541.

\section{Results}

Table 1 shows the demographic data of the 3,261 patients included in the present study, while Table 2 shows the success rate of $H$. pylori eradication therapy. The eradication rate of the firstline clarithromycin-based triple therapy with PPIs was significantly lower than that with PCAB $(p<0.001$, in ITT and PP analyses). However, the rate of the second-line metronidazolebased triple therapy did not differ significantly between the PPI and PCAB groups.

The eradication rate of the first-line therapy with PPIs was significantly lower for the young to middle-aged group than for the elder group $(p<0.01$ for ITT and $p<0.001$ for PP). In contrast, for the first-line therapy with PCAB and the second-line metronidazole-based triple therapy, the eradication rates were similar in both age groups.

The eradication rates of clarithromycin-based triple therapy with PPIs were increased stepwise in young ( $\leq 30$ years), middleaged ( $30-50$ years), and old patients ( $>50$ years) (Fig. 1$)$.

The data of side effects were available in 917 patients. The incidence of side effects was $5.7 \%(30 / 529)$ in patients receiving the first-line clarithromycin-based triple therapy with PPIs, and
$8.4 \%(13 / 154)$ in patients receiving the first-line clarithromycinbased triple therapy with PCAB. The incidence of side effects was $3.3 \%(6 / 184)$ in patients receiving the second-line metronidazolebased triple therapy with PPIs, and 6\% (3/50) in patients receiving the second-line metronidazole-based triple therapy with PCAB. For the incidence of side effects, there was no significant difference between PPIs and PCAB. In these cases, the side effects were mild and mainly included diarrhea, skin rash, or stomatitis.

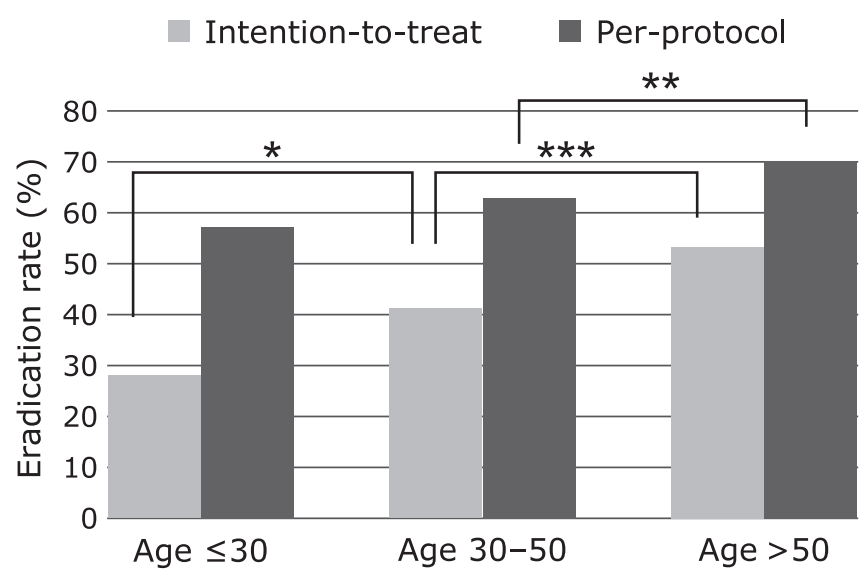

Fig. 1. The eradication rates of clarithromycin-based triple therapy with PPIs in young ( $\leq 30$ years), middle-aged ( $30-50$ years), and old patients ( $>50$ years). ${ }^{*} p<0.05,{ }^{* *} p<0.01,{ }^{* *} p<0.001$.

Table 1. Demographic data of patients undergoing eradication therapy for $\mathrm{H}$. pylori

\begin{tabular}{llccc}
\hline & \multicolumn{1}{c}{ Regimen } & Number & Age & Sex (M/F) \\
\hline Total & Triple therapy & 3,261 & $52.3 \pm 7.5$ & $1,508 / 1,753$ \\
\hline First-line treatment & $\begin{array}{l}\text { Lansoprazole or rabeprazole } \\
\text { Clarithromycin, amoxicillin }\end{array}$ & 2,173 & $52.7 \pm 13.8$ & $1,012 / 1,161$ \\
\cline { 2 - 5 } & $\begin{array}{l}\text { Vonoprazan } \\
\text { Clarithromycin, amoxicillin }\end{array}$ & 353 & $50.4 \pm 13.3$ & $138 / 215$ \\
\hline Second-line treatment & $\begin{array}{l}\text { Lansoprazole or rabeprazole } \\
\text { Metronidazole, amoxicillin }\end{array}$ & 650 & $51.7 \pm 13.0$ & $322 / 328$ \\
\hline & $\begin{array}{l}\text { Vonoprazan } \\
\text { Metronidazole, amoxicillin }\end{array}$ & 85 & $53.5 \pm 13.3$ & $36 / 49$ \\
\hline
\end{tabular}

M, male; F, female.

Table 2. Success rate of $H$. pylori eradication therapy

\begin{tabular}{lllll}
\hline & \multicolumn{1}{c}{ Regimen } & Age & \multicolumn{1}{c}{ Per protocol } & \multicolumn{1}{c}{ Intention to treat } \\
\hline First-line treatment & Lansoplazole or rabeprazole & All & $66.8 \%(1,024 / 1,532)$ & $47.1 \%(1,024 / 2,173)$ \\
& Clarithromycin & $\leq 50$ & $62.6 \%(403 / 644)$ & $40.1 \%(403 / 1,006)$ \\
& Amoxicillin & $>50$ & $69.9 \%(621 / 888)^{\#}$ & $53.2 \%(621 / 1,167)^{\# \#}$ \\
\cline { 2 - 4 } & Vonoprazan & All & $89.4 \%(220 / 246)^{* *}$ & $62.3 \%(220 / 353)^{* *}$ \\
& Clarithromycin & $\leq 50$ & $89.4 \%(118 / 132)^{* *}$ & $60.5 \%(118 / 195)^{* *}$ \\
& Amoxicillin & $>50$ & $89.5 \%(102 / 114)^{* *}$ & $64.5 \%(102 / 158)^{*}$ \\
\hline Second-line treatment & Lansoprazole or rabeprazole & All & $90.5 \%(479 / 529)$ & $73.7 \%(479 / 650)$ \\
& Metronidazole & $\leq 50$ & $90.8 \%(216 / 238)$ & $68.4 \%(216 / 316)$ \\
& Amoxicillin & $>50$ & $90.4 \%(263 / 291)$ & $78.7 \%(263 / 334)$ \\
\cline { 2 - 4 } & Vonoprazan & All & $96.8 \%(61 / 63)$ & $71.8 \%(61 / 85)$ \\
& Metronidazole & $\leq 50$ & $96.8 \%(30 / 31)$ & $78.9 \%(30 / 38)$ \\
& Amoxicillin & $>50$ & $96.9 \%(31 / 32)$ & $66.0 \%(31 / 47)$ \\
\hline
\end{tabular}

${ }^{\#} p<0.01,{ }^{\# \#} p<0.001$, Age $>50$ group vs age $\leq 50$ group. ${ }^{*} p<0.01,{ }^{* *} p<0.001$, Vonoprazan based regimen vs lansoplazole or rabeprazole based regi-
men. 


\section{Discussion}

The results of the present study showed that first-line clarithromycin-based triple therapy with PPIs demonstrated a significantly lower eradication rate than the same therapy with PCAB. Further, clarithromycin-based triple therapy with PPIs failed significantly more frequently in young to middle-aged patients (age $\leq 50$ years) than in older patients (age $<50$ years). However, age had no impact on the eradication rate for PCABbased therapy or metronidazole-based therapy.

In their 2008-2009 study, Okamura et al. ${ }^{(17)}$ reported that the rates of clarithromycin resistance were $51.6 \%, 46.8 \%$, and $25.5 \%$ in young ( $\leq 30$ years), middle-aged ( $30-50$ years), and old patients $(>50$ years). As indicated by these values, the rate of resistance was significantly higher in the young $(p=0.03)$ and middle-aged $(p<0.01)$ patients than the old patients. However, metronidazole resistance was similar among the three groups.

We believe that the higher rate of clarithromycin resistance in young to middle-aged group adversely affected the success rate of clarithromycin-based triple therapy with PPIs. However, surprisingly, this higher resistance rate did not affect the success rate of clarithromycin-based triple therapy with $\mathrm{PCAB}$.

Murakami et al. ${ }^{(14)}$ reported that the eradication rate of first-line clarithromycin-based triple therapy was significantly higher with vonoprazan than with lansoprazole in patients infected with clarithromycin-resistant strains of $H$. pylori $(82.0 \%$ vs $40.0 \%$; $p<0.0001)$. Vonoprazan may compensate for clarithromycin resistance, but the underlying mechanism remains unclear. One explanation could be synergistic actions between vonoprazan and the antimicrobials used, in that $H$. pylori is more susceptible to antimicrobials when its replicative capability is restored at $\mathrm{pH}$ values exceeding 6 , which are the levels facilitated by vonoprazan. ${ }^{(18)}$

The present study has some limitations. First, many patients did not return to the clinic to undergo a urea breath test for evaluating the results of eradication therapy because of routine clinical practice. Yogeswaran et al. ${ }^{(19)}$ also reported that confirmation of eradication was lacking in a significant proportion of patients in clinical practice. Second, this study was retrospective, and adverse

\section{References}

1 Asaka M, Kato M, Graham DY. Strategy for eliminating gastric cancer in Japan. Helicobacter 2010; 15: 486-490.

2 Suzuki H, Nishizawa T, Hibi T. Helicobacter pylori eradication therapy. Future Microbiol 2010; 5: 639-648.

3 Nishizawa T, Suzuki H. Gastric carcinogenesis and underlying molecular mechanisms: Helicobacter pylori and novel targeted therapy. Biomed Res Int 2015; 2015: 794378.

4 Naito Y, Yoshikawa T. Molecular and cellular mechanisms involved in Helicobacter pylori-induced inflammation and oxidative stress. Free Radic Biol Med 2002; 33: 323-336.

5 Suzuki M, Suzuki H, Minegishi Y, Ito K, Nishizawa T, Hibi T. H. pylorieradication therapy increases RUNX3 expression in the glandular epithelial cells in enlarged-fold gastritis. J Clin Biochem Nutr 2010; 46: 259-264.

6 Handa O, Naito Y, Yoshikawa T. Helicobacter pylori: a ROS-inducing bacterial species in the stomach. Inflamm Res 2010; 59: 997-1003.

7 Sasaki M, Ogasawara N, Utsumi K, et al. Changes in 12-year first-line eradication rate of Helicobacter pylori based on triple therapy with proton pump inhibitor, amoxicillin and clarithromycin. J Clin Biochem Nutr 2010; 47: 53-58.

8 Nishizawa T, Suzuki H, Suzuki M, Takahashi M, Hibi T. Proton pump inhibitor-amoxicillin-clarithromycin versus proton pump inhibitor-amoxicillinmetronidazole as first-line Helicobacter pylori eradication therapy. J Clin Biochem Nutr 2012; 51: 114-116.

9 Nishizawa T, Maekawa T, Watanabe N, et al. Clarithromycin versus metronidazole as first-line Helicobacter pylori eradication: a multicenter, prospective, randomized controlled study in Japan. J Clin Gastroenterol 2015; 49: 468-471.

10 Hirata K, Suzuki H, Nishizawa T, et al. Contribution of efflux pumps to events were partially available. A prospective follow-up study should be performed to confirm our results.

In conclusion, the PPI-clarithromycin-amoxicillin regimen showed a significantly lower $H$. pylori eradication rate than the PCAB-clarithromycin-amoxicillin regimen did. Additionally, with the former, the eradication rate in the young to middle-aged group was significantly lower than that in the older group of patients. Thus, with clarithromycin-based triple therapy, PCAB is a better choice of antisecretory agent than PPIs, especially in young to middle-aged patients (age $\leq 50$ years).

\section{Conflict of Interest}

During the last two years, HS received scholarship funds for the research from Daiichi-Sankyo Co., Ltd., Otsuka Pharmaceutical Co., Ltd., Shionogi Pharm. Co., Ltd., Takeda Pharmaceutical Co., Ltd., Toshiba Co., Ltd. and Tsumura Co., Ltd., and received service honoraria from Astellas Pharm Inc., Astra-Zeneca K.K., EA Pharma. Co., Ltd., Mylan EPD Co., Ltd., Otsuka Pharmaceutical Co., Ltd., Takeda Pharmaceutical Co., Ltd., Tsumura Co., Ltd., and Zeria Pharmaceutical Co., Ltd. TK received scholarship funds for the research from Astellas Pharm Inc., Astra-Zeneca K.K., Otsuka Pharmaceutical Co., Ltd., Takeda Pharmaceutical Co., Ltd., Eisai Pharmaceutical Co., Ltd., Zeria Pharmaceutical Co., Ltd., Tanabe Mitsubishi Pharmaceutical Co., Ltd., JIMRO Co., Ltd., Kyorin Pharmaceutical Co., Ltd., and received service honoraria from Astellas Pharm Inc., Eisai Pharmaceutical Co., Ltd., JIMRO Co., Ltd., Tanabe Mitsubishi Pharmaceutical Co., Ltd., Otsuka Pharmaceutical Co., Ltd., Takeda Pharmaceutical Co., Ltd., Miyarisan Pharmaceutical Co., Ltd., and Zeria Pharmaceutical Co., Ltd. NY received scholarship funds for the research from Takeda Pharmaceutical Co., Ltd., Eisai Co., Ltd., Kaigen Pharm Co., Ltd., Boston Scientific Japan K.K., Nihon Pharmaceutical Co., Ltd., Hoya corporation and Otsuka Pharmaceutical Co., Ltd. The funding source had no role in the design, practice or analysis of this study. There are no other conflicts of interests for this article. clarithromycin resistance in Helicobacter pylori. J Gastroenterol Hepatol 2010; 25 Suppl 1: S75-S79.

11 Nishizawa T, Suzuki H, Takahashi M, Suzuki M, Hibi T. Delay of secondline eradication therapy for Helicobacter pylori can increase eradication failure. J Gastroenterol Hepatol 2013; 28: 1608-1610.

12 Nishizawa T, Suzuki H, Masaoka T, Iwasaki E, Hibi T. A new eradication resistance index as a predictor of metronidazole-containing second-line treatment of Helicobacter pylori. Digestion 2007; 76: 215-220.

13 Nishizawa T, Suzuki H, Hibi T. Quinolone-based third-line therapy for Helicobacter pylori eradication. J Clin Biochem Nutr 2009; 44: 119-124.

14 Murakami K, Sakurai Y, Shiino M, Funao N, Nishimura A, Asaka M. Vonoprazan, a novel potassium-competitive acid blocker, as a component of first-line and second-line triple therapy for Helicobacter pylori eradication: a phase III, randomised, double-blind study. Gut 2016; 65: 1439-1446.

15 Mamori S, Higashida A, Kawara F, et al. Age-dependent eradication of Helicobacter pylori in Japanese patients. World J Gastroenterol 2010; 16: 4176-4179.

16 Nishizawa T, Suzuki H, Arano T, et al. Characteristics of gastric cancer detected within 1 year after successful eradication of Helicobacter pylori. $J$ Clin Biochem Nutr 2006; 59: 226-230.

17 Okamura T, Suga T, Nagaya T, et al. Antimicrobial resistance and characteristics of eradication therapy of Helicobacter pylori in Japan: a multigenerational comparison. Helicobacter 2014; 19: 214-220.

18 Nishizawa T, Suzuki H, Maekawa T, et al. Dual therapy for third-line Helicobacter pylori eradication and urea breath test prediction. World $J$ Gastroenterol 2012; 18: 2735-2738.

19 Yogeswaran K, Chen G, Cohen L, et al. How well is Helicobacter pylori treated in usual practice? Can J Gastroenterol 2011; 25: 543-546. 